\title{
TRACING THE BIPOLAR OUTFLOW FROM ORION SOURCE I
}

\author{
R. L. Plambeck ${ }^{1}$, M. C. H. Wright ${ }^{1}$, D. N. Friedel ${ }^{2}$, S. L. Widicus Weaver ${ }^{3}$, A. D. Bolatto ${ }^{4}$, M. W. Pound ${ }^{4}$, D. P. Woody ${ }^{5}$, \\ J. W. LAMB ${ }^{5}$, AND S. L. SCOTT ${ }^{5}$ \\ ${ }^{1}$ Radio Astronomy Laboratory, 601 Campbell Hall, University of California, Berkeley, CA 94720, USA \\ ${ }^{2}$ Department of Astronomy, 1002 W. Green St., University of Illinois, Urbana, IL 61801, USA \\ ${ }^{3}$ Department of Chemistry, Emory University, Atlanta, GA 30322, USA \\ ${ }^{4}$ Department of Astronomy, University of Maryland, College Park, MD 20742, USA \\ ${ }^{5}$ Owens Valley Radio Observatory, California Institute of Technology, P.O. Box 968, Big Pine, CA 93513, USA \\ Received 2009 July 10; accepted 2009 September 9; published 2009 September 23
}

\begin{abstract}
Using CARMA, we imaged the $87 \mathrm{GHz} \mathrm{SiO} v=0 J=2-1$ line toward Orion-KL with 0'45 angular resolution. The maps indicate that radio source I drives a bipolar outflow into the surrounding molecular cloud along a NE-SW axis, in agreement with the model of Greenhill et al. The extended high-velocity outflow from Orion-KL appears to be a continuation of this compact outflow. High-velocity gas extends farthest along a NW-SE axis, suggesting that the outflow direction changes on timescales of a few hundred years.
\end{abstract}

Key words: ISM: individual (Orion-KL) - ISM: jets and outflows - masers - stars: formation

\section{INTRODUCTION}

Bipolar outflows are ubiquitous from young low-mass stars, but are difficult to observe toward high-mass stars, which typically form in crowded, physically complex regions that are quickly disrupted by ionization fronts and stellar winds.

The nearest region of massive star formation is the Kleinmann-Low Nebula in Orion, at a distance of about $400 \mathrm{pc}$ (Sandstrom et al. 2007; Menten et al. 2007). Measurements of $\mathrm{H}_{2} \mathrm{O}$ maser proper motions led Genzel et al. (1981) to suggest that two distinct outflows originate from this region-a low-velocity $\left(18 \mathrm{~km} \mathrm{~s}^{-1}\right)$ outflow along a NE-SW axis and a high-velocity (30-100 $\mathrm{km} \mathrm{s}^{-1}$ ) flow extending roughly NW-SE. Both outflows were inferred to originate within a few arcseconds of radio source I, a young star with a luminosity of $10^{4}-10^{5} L_{\odot}$ (Gezari et al. 1998). The high-velocity outflow also manifests itself as lobes of shock-excited $\mathrm{H}_{2}$ (Beckwith et al. 1978) and as broad, weakly bipolar line wings in $\mathrm{CO}$ and other molecules (Zuckerman et al. 1976; Kwan \& Scoville 1976; Erickson et al. 1982; Chernin \& Wright 1996).

Source I is associated with a cluster of $\mathrm{SiO}$ masers. From fits to $2^{\prime \prime}$ resolution BIMA data, Plambeck et al. (1990) found that the $J=2-1 v=1 \mathrm{SiO}$ masers were clustered along two arcs offset $\sim 0^{\prime \prime} 08 \mathrm{NW}$ and SE of source I. Plambeck et al. (1990) reproduced this pattern with a model of maser emission from a rotating, expanding disk, tilted at $45^{\circ}$ to the plane of the sky. The axis of the model disk was projected at P.A. $145^{\circ}$, suggesting that the high-velocity outflow emerged along its poles.

Later observations showed that the $J=2-1 \mathrm{SiO}$ line in the $v=0$ vibrational level also was masing (Wright et al. 1995) in a 1000 AU long hourglass-shaped region centered on source I. Because the hourglass was elongated NE-SW, it was natural to associate it with the outer regions of the model $v=1$ maser disk. SiO line widths were $\sim 30 \mathrm{~km} \mathrm{~s}^{-1}$ across the entire hourglass, inconsistent with Keplerian rotation, so Wright et al. (1995) suggested that the emission traced the turbulent boundary layer between an underlying, unseen, disk and the high-velocity outflow.

More recent observations have cast doubt on this picture. Very Long Baseline Array (VLBA) observations of the $43 \mathrm{GHz} v=1$ SiO masers (Greenhill et al. 1998; Doeleman et al. 1999) showed that the brightest maser spots were clustered in four groups, rather than in a ring as predicted by the Plambeck et al. (1990) model. A bridge of emission connecting the two southern clusters led Greenhill et al. (2004) to propose that the $v=1$ masers originate in a nearly edge-on disk rotating about a NE-SW axis, perpendicular to the previously hypothesized disk, and that the $v=0 \mathrm{SiO}$ emission traces the base of the $18 \mathrm{~km} \mathrm{~s}^{-1}$ outflow. This model did not attempt to explain the high-velocity outflow.

Proper-motion measurements of source I (Rodríguez et al. 2005; Gómez et al. 2008) also pose problems for the original model. These data indicate that source I is moving to the SE at $0^{\prime}$.007 year ${ }^{-1}$, plowing through the molecular cloud at $\sim 14 \mathrm{~km} \mathrm{~s}^{-1}$. In that case, a $1000 \mathrm{AU}$ diameter disk would rapidly be stripped away by the ram pressure of the ambient gas unless source I were implausibly massive.

In this Letter, we report 0!45 (180 AU) resolution images of the $\mathrm{SiO} v=0 J=2-1$ line obtained with CARMA. The new maps provide the best evidence to date that the $v=0 \mathrm{SiO}$ emission originates in a NE-SW outflow from source I, as in the Greenhill et al. (2004) model. The maps also suggest that the high-velocity outflow is a continuation of this compact flow.

\section{OBSERVATIONS}

Observations were made with CARMA in the A-, B-, and Carrays, providing projected antenna spacings ranging from 5 to $545 \mathrm{k} \lambda$. The total integration time on Orion-KL was $3.4 \mathrm{hr}$ in the A-array (2009 January), $3.7 \mathrm{hr}$ in the B-array (2008 February), and $5 \mathrm{hr}$ in the C-array (2009 May).

The correlator was configured for simultaneous observations of the $J=2-1 \mathrm{SiO}$ transitions in both the $v=0(86.847 \mathrm{GHz})$ and $v=1(86.243 \mathrm{GHz})$ vibrational levels. For each transition, the velocity coverage was $104 \mathrm{~km} \mathrm{~s}^{-1}$ and the resolution was $3.4 \mathrm{~km} \mathrm{~s}^{-1}$ after Hanning smoothing. Observations of quasars were used to calibrate phase and amplitude ripples across the I.F. passband. After these were removed, the visibility data were self-calibrated using a $28 \mathrm{~km} \mathrm{~s}^{-1}$ wide channel that included all the bright $v=1$ maser features. Only baselines shorter than $250 \mathrm{k} \lambda$ were used in the self-calibration because the maser is slightly resolved on the longest baselines. The integration time per data record was $4 \mathrm{~s}$ in the A-array and $10 \mathrm{~s}$ in the B-and $\mathrm{C}$-arrays, allowing near-complete removal of atmospheric phase 
fluctuations; phase residuals on the maser were typically $\pm 1^{\circ}$ on all baselines after self-calibration.

Maps of the $v=0 \mathrm{SiO}$ line were made with the Miriad data reduction package. Uniformly weighting the visibility data yielded a $0.54 \times 0 . \prime 40$ synthesized beam at P.A. $52^{\circ}$. Because the data were self-calibrated using the $v=1 \mathrm{SiO}$ masers, the maps are centered on the intensity-weighted mean maser position, which does not coincide precisely with radio source I. An $86 \mathrm{GHz}$ continuum map made with A-array data from 2009 January showed that source I was centered $0.01 \pm 0$.'01 W and $0.03 \pm 00^{\prime} 01 \mathrm{~S}$ of the mean $v=1$ maser position. We adjusted the central coordinate of the maps to place source I at $05^{\mathrm{h}} 35^{\mathrm{m}} 14.515,-5^{\circ} 22^{\prime} 30^{\prime} \cdot 57$, as derived from VLA propermotion data (Gómez et al. 2008).

\section{RESULTS}

Figure 1 presents a series of channel maps showing the $v=0$ $\mathrm{SiO}$ emission in an $8^{\prime \prime} \times 8^{\prime \prime}$ box centered on source I. The lowest contour on these images is $75 \mathrm{~K}$ and the peak is $3800 \mathrm{~K}$.

Although the maps in Figure 1 are consistent with those published by Wright et al. (1995) and Chandler \& de Pree (1995), the higher quality CARMA images clearly indicate that $v=0 \mathrm{SiO}$ emission originates in a NE-SW bipolar outflow from source I, as in the Greenhill et al. (2004) model. The central channels show the limb-brightened edges of two cones centered on source I; emission in the line wings is brightest interior to these cones. Although the outflow appears to lie nearly in the plane of the sky, there is a measurable red-blue asymmetry: redshifted gas at $V_{\mathrm{LSR}}=22 \mathrm{~km} \mathrm{~s}^{-1}$ appears as a narrow jet to the $\mathrm{SW}$, while blueshifted gas at $V_{\mathrm{LSR}}=-9 \mathrm{~km} \mathrm{~s}^{-1}$ is offset to the NE. This asymmetry is inconsistent with the old model in which the emission originates in the turbulent boundary layer of a disk.

It is clear that the $\mathrm{SiO}$ outflow corresponds to the $18 \mathrm{~km} \mathrm{~s}^{-1}$ $\mathrm{H}_{2} \mathrm{O}$ maser outflow identified by Genzel et al. (1981): toward source I the $\mathrm{SiO}$ line has a full width at zero intensity of $\sim 36 \mathrm{~km} \mathrm{~s}^{-1}$, and both $\mathrm{SiO}$ and $\mathrm{H}_{2} \mathrm{O}$ masers extend along a NE$\mathrm{SW}$ axis. It is likely, however, that this material was accelerated by a much faster wind. Source $I$ is traveling to the SE at $14 \mathrm{~km} \mathrm{~s}^{-1}$ in the plane of the sky (Gómez et al. 2008), so gas moving outward at only $18 \mathrm{~km} \mathrm{~s}^{-1}$ would appear to be swept back into an arc with arms trailing to the NW.

The high signal-to-noise level in the maps, exceeding 100:1 on the bright maser spots, makes it feasible to enhance the resolution of the innermost region of the outflow. One can fit the position of an isolated maser feature to an accuracy of $\sim 0.5 \theta_{\text {FWHM }} /$ SNR (Reid et al. 1988), where $\theta_{\text {FWHM }}$ is the apparent FWHM of the source and SNR is the signal-tonoise ratio. Here, the maser spots overlap heavily, however, so fitting their positions individually is difficult. Instead, we CLEANed the maps in the usual way, then convolved the CLEAN components with a $0^{\prime \prime} 25$ FWHM Gaussian restoring beam, about half the width of the synthesized beam. Figure 2 shows the resulting $\mathrm{SiO} v=0$ image at $V_{\mathrm{LSR}}=5 \mathrm{~km} \mathrm{~s}^{-1}$ in the $2^{\prime \prime} \times 2^{\prime \prime}$ central region. Red contours show the $229 \mathrm{GHz}$ continuum emission from source I, which is centered in the waist of the $\mathrm{SiO}$ hourglass; $\mathrm{SiO}$ masers in the $v=1$ vibrational level are clustered along the edges of the continuum source.

The brightest $v=0 \mathrm{SiO}$ masers lie along two bars offset $\sim 0$ '.5 NE and SW of the continuum source. The ends of the bars are limb brightened, suggesting that the masers originate in two annuli. Position-velocity cuts through the bars, shown in panels (a) and (b), hint that these annuli are expanding radially at $\sim 10 \mathrm{~km} \mathrm{~s}^{-1}$, as modeled by the green ellipses.

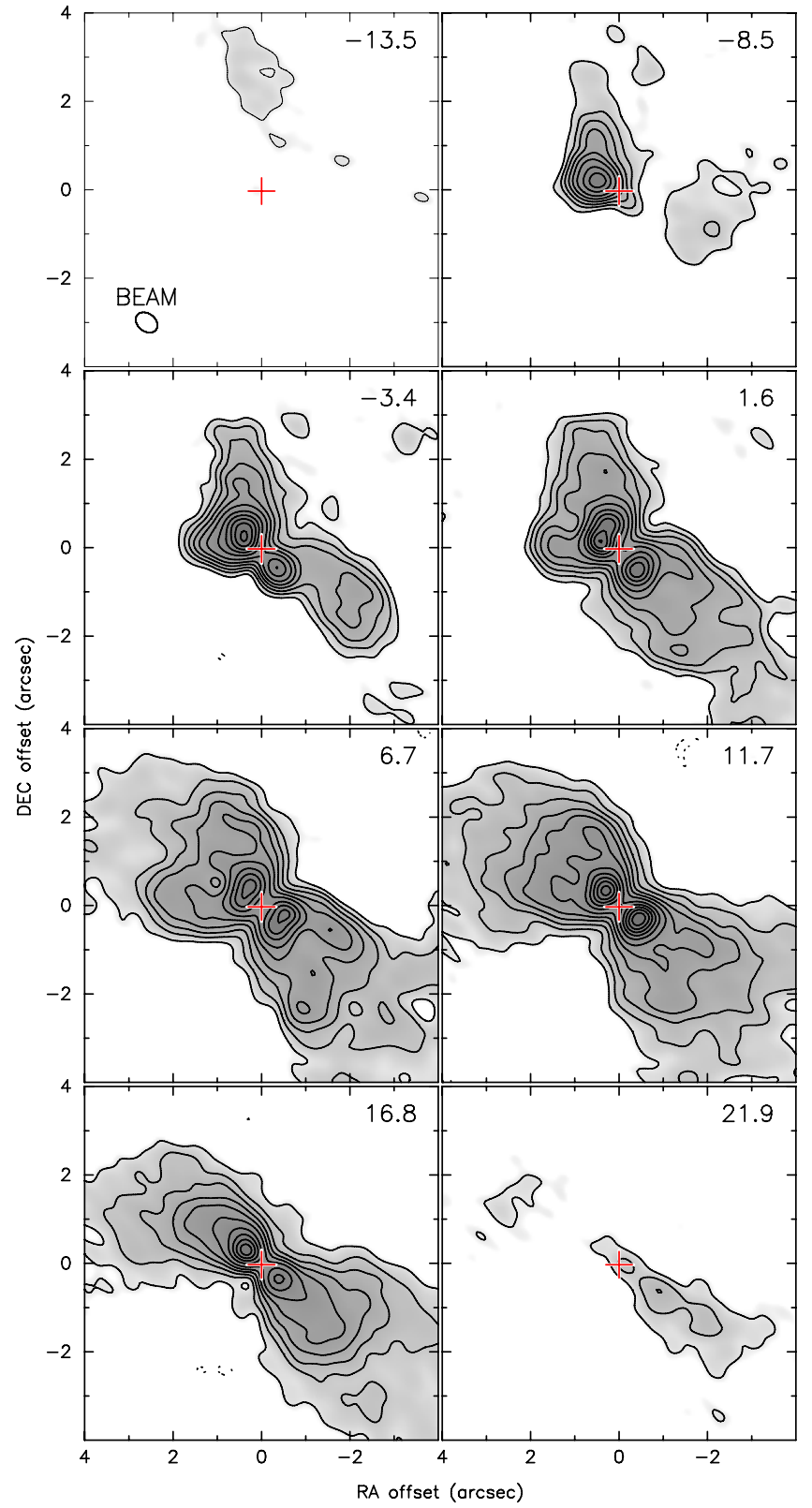

Figure 1. Channel maps, $5.06 \mathrm{~km} \mathrm{~s}^{-1}$ wide, of the $\mathrm{SiO} v=0 J=2-1$ line toward Orion-KL. Each box is $8^{\prime \prime}$ on a side and is labeled with the center LSR velocity. A cross marks the location of radio source I. The contour levels are $\pm 75,150,250,400,600 \mathrm{~K}$, then $900-3400 \mathrm{~K}$ in $500 \mathrm{~K}$ steps; $745 \mathrm{~K}=1 \mathrm{Jy}$ beam $^{-1}$. The noise level is $12 \mathrm{~K}$, increasing to $19 \mathrm{~K}$ in the center channels due to limited dynamic range.

What about the high-velocity outflow? In order to obtain higher sensitivity for extended emission, we tapered the weighting of the visibility data to produce a set of channel maps with a 1". $13 \times 0$ 0'.93 synthesized beam. The rms noise is $4 \mathrm{~K}$, increasing to as much as $8 \mathrm{~K}$ in channels with strong maser emission due to limited dynamic range. Figure 3 shows the full extent of the $\mathrm{SiO}$ emission detected in these maps, color coded to indicate the intensity-weighted LSR velocity. Brightness temperatures outside the central $8^{\prime \prime} \times 5^{\prime \prime}$ hourglass are $<100 \mathrm{~K}$, hence this emission is likely to be thermal. As in previous maps of the high-velocity outflow (Chernin \& Wright 1996), the strongest redshifted emission is E of source I, while blueshifted gas is offset to the NW.

Figure 3 suggests that the extended high-velocity outflow is simply a continuation of the compact outflow. For example, 

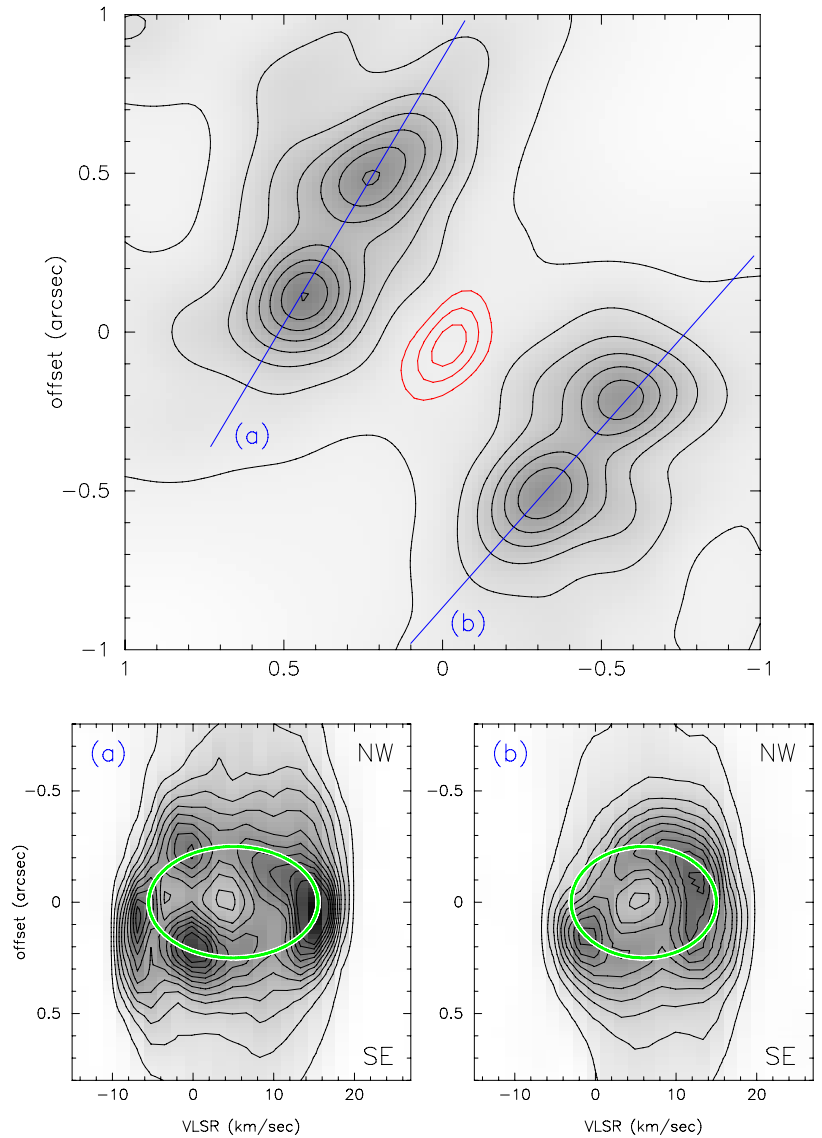

Figure 2. Top: $\mathrm{SiO} v=0$ map in a $10 \mathrm{~km} \mathrm{~s}^{-1}$ wide channel centered at $V_{\mathrm{LSR}}=$ $5 \mathrm{~km} \mathrm{~s}^{-1}$, deconvolved to $0^{\prime} \cdot 25$ resolution as described in the text. The contour interval is $780 \mathrm{~K}\left(0.3 \mathrm{Jy}_{\text {beam }}{ }^{-1}\right)$, the rms noise is $80 \mathrm{~K}$, and the peak intensity is $6250 \mathrm{~K}$. Red contours show the $229 \mathrm{GHz}$ radio continuum from source I, measured at CARMA with a 0"' 15 synthesized beam (R. L. Plambeck et al. 2009, in preparation). Bottom: position-velocity cuts along lines (a) and (b) generated from $3.4 \mathrm{~km} \mathrm{~s}^{-1}$ resolution channel maps, also deconvolved to 0.25 resolution. The contour spacing is $780 \mathrm{~K}$; the rms noise of the channel maps ranges from 50 to $140 \mathrm{~K}$. Green ellipses show velocities expected for a 0.5 diameter ring expanding radially at $10.5 \mathrm{~km} \mathrm{~s}^{-1}$ in panel (a) and $9 \mathrm{~km} \mathrm{~s}^{-1}$ in panel (b).

$\sim 3^{\prime \prime}$ NE of source I the compact outflow twists to the E and points directly toward some of the most redshifted gas in the extended flow. The opening angle appears to broaden abruptly at this point, perhaps as the outflow breaks out of dense gas surrounding source I. Two HH objects, 152-228 and 152-229, lie at the tip of the flow; proper-motion measurements indicate that they are moving away from source I at $35-50 \mathrm{~km} \mathrm{~s}^{-1}$ (Doi et al. 2002).

Figure 3 suggests that $\mathrm{SiO}$ velocities increase away from source I. This is particularly evident in the redshifted gas, as shown in Figure 4; note the absence of high-velocity gas within $4^{\prime \prime}$ of the star. There are several possible explanations for these velocity gradients.

First, $\mathrm{SiO}$ emission may originate in dense clumps that are entrained in a fast stellar wind. The wind continues to accelerate the clumps as they move outward. Evidence for such acceleration is relatively common in outflows (Stahler 1994).

Second, it is possible that the $\mathrm{SiO}$ emitting gas was ejected by an explosive event that flung out material with a range of speeds. The fastest-moving ejecta now are farthest from the center. The system of shock-excited $\mathrm{H}_{2}$ fingers in Orion appears to have been created by such an explosive event (Allen \& Burton 1993; Doi et al. 2002), perhaps as a swept-up shell was fragmented by a faster-moving wind (Stone et al. 1995).

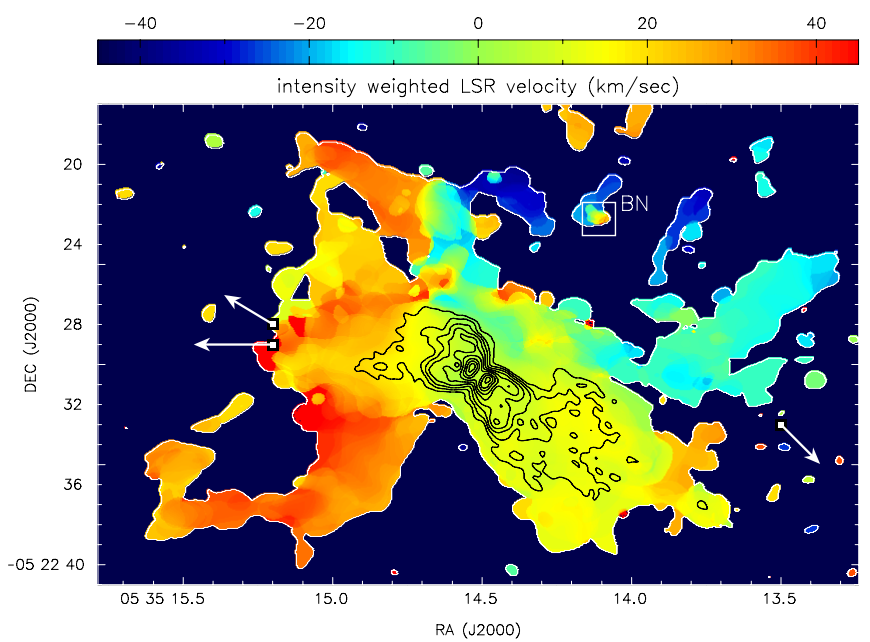

Figure 3. Velocity moment map of the $v=0 \quad J=2-1 \mathrm{SiO}$ line derived from $1^{\prime \prime}$ resolution channel maps covering the velocity range $-30<V_{\mathrm{LSR}}<47 \mathrm{~km} \mathrm{~s}^{-1}$. Pixels were averaged into the moment map only if they exceeded $3 \times$ the rms noise, which ranged from 4 to $8 \mathrm{~K}$ across the channels. Black contours show the $V_{\mathrm{LSR}}=6.7 \mathrm{~km} \mathrm{~s}^{-1}$ channel map from Figure 1, with the same contour intervals. A white box marks the location of the Becklin-Neugebauer Object; radio continuum from $\mathrm{BN}$ corrupts the velocity moment map in this direction. White arrows show proper motions of the HH objects 152-228, 152-229, and 135-233 over 150 years (Doi et al. 2002).

Third, it is possible that the extended gas provides a fossil record of a precessing outflow. Currently, the outflow axis is $\mathrm{NE}-\mathrm{SW}$, with the (redshifted) SW lobe tipped slightly into the plane of the sky. Approximately $10^{\prime \prime}$ from the star, the outflow axis appears to be E-W, with the E lobe tipped into the plane of the sky. If the true outflow velocity is $100 \mathrm{~km} \mathrm{~s}^{-1}$, this $>45^{\circ}$ change in direction took place in 200 years. Precession occurs because of tidal interactions in noncoplanar binary systems; periods of a few thousand years are typical of systems with binary separations of tens of AU (Terquem et al. 1999). A secondary in an eccentric orbit can change both the outflow direction and the mass-loss rate periodically, as in the model of CepA presented by Cunningham et al. (2009).

Zapata et al. (2009) report that much of the emission from the high-velocity $\mathrm{CO}$ line wings in Orion originates from filamentary structures similar to the $\mathrm{H}_{2}$ fingers, with linear velocity gradients along them. Zapata et al. (2009) argue that the high-velocity flow is not a classical stellar outflow, but instead was produced by the disintegration, about 500 years ago, of a multiple stellar system that contained source I and $\mathrm{BN}$. While this scenario is consistent with the velocity gradients evident in Figure 4 and with the vaguely filamentary character of the $\mathrm{SiO}$ emission away from source I, it implies that the apparently continuous transition from the $18 \mathrm{~km} \mathrm{~s}^{-1}$ flow to the high-velocity outflow seen in Figure 3 is illusory. We are reluctant to accept that conclusion, and suggest that perhaps the disintegration of the multiple system triggered an abrupt increase in the velocity or mass-loss rate of the outflow from source I, creating the finger system as in the model of Stone et al. (1995).

\section{CONCLUSIONS}

New maps of the $\mathrm{SiO}$ line in the $v=0 J=2-1$ transition toward Orion-KL provide some of the clearest evidence to date that radio source I, thought to be a massive young star, drives a bipolar outflow into the surrounding molecular cloud along a NE-SW axis. This outflow contains multiple water masers and 


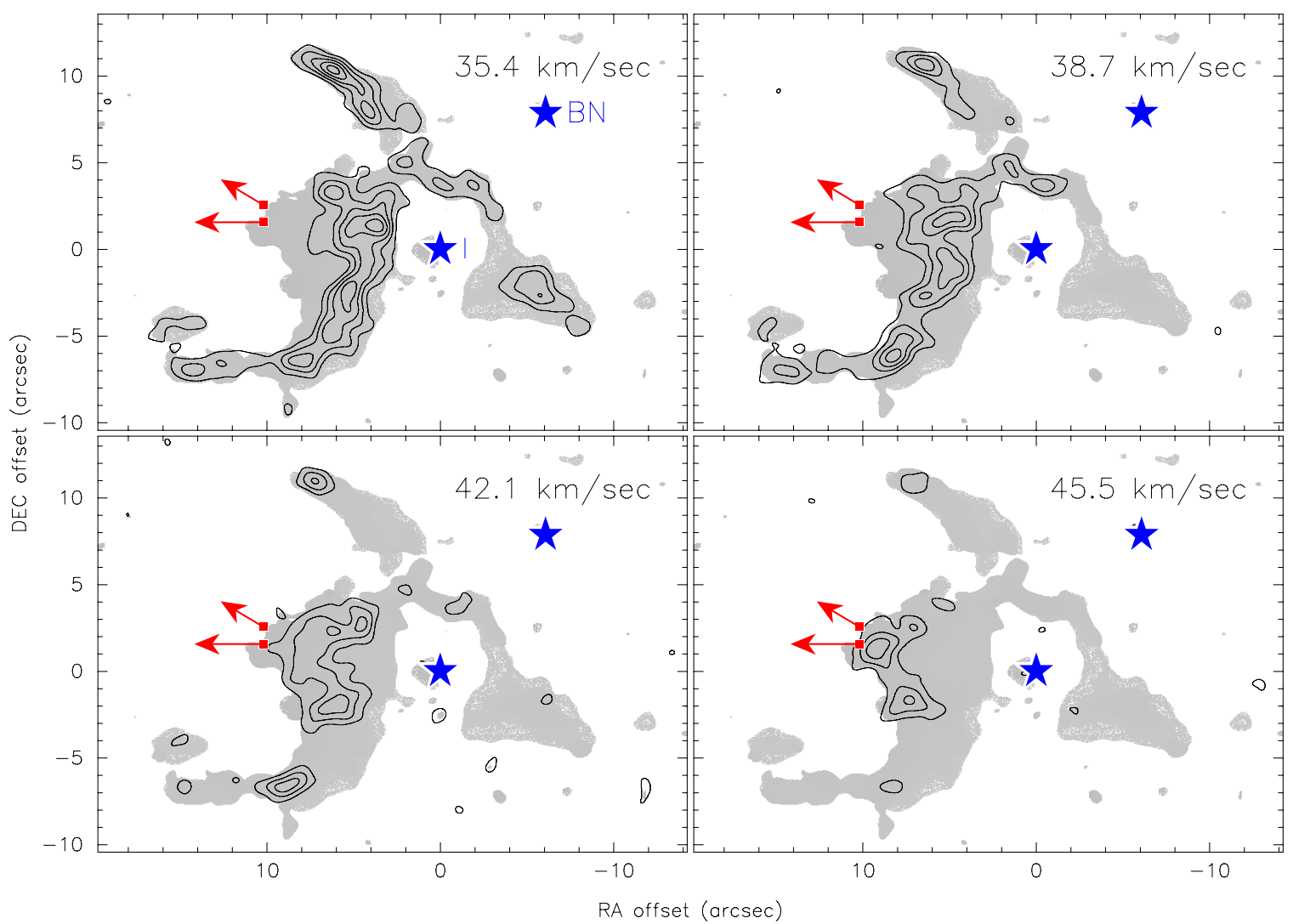

Figure 4. $\mathrm{SiO} v=0$ channel maps showing a velocity gradient in the redshifted lobe of the high-velocity outflow. The contour interval is $12 \mathrm{~K}$; the rms noise is $4 \mathrm{~K}$. The $V_{\mathrm{LSR}}$ of each channel is indicated. Gray shading indicates pixels for which there is $3 \sigma$ emission in at least one channel in the velocity range $29<V_{\mathrm{LSR}}$ $<52 \mathrm{~km} \mathrm{~s}^{-1}$. Blue stars mark the positions of source I and BN. Red arrows show proper motions of the HH objects 152-228 and 152-229.

conventionally is referred to as the $18 \mathrm{~km} \mathrm{~s}^{-1}$ flow. The outflow velocity must be substantially greater than $18 \mathrm{~km} \mathrm{~s}^{-1}$, however, or the outflow lobes would trail back to the NW owing to the proper motion of source I. Close to source I, the $\mathrm{SiO} v=0$ emission is masing. The strongest masers are clustered in two annuli offset $\sim 200 \mathrm{AU}$ from source I along the central axis of the outflow.

More extended, higher velocity gas extends along a NW-SE axis, almost perpendicular to the $18 \mathrm{~km} \mathrm{~s}^{-1}$ flow. The $\mathrm{SiO}$ maps suggest that this weakly bipolar outflow is an extension of the $18 \mathrm{~km} \mathrm{~s}^{-1}$ flow, which may change direction on timescales of a few hundred years.

Support for CARMA construction was derived from the states of California, Illinois, and Maryland, the Gordon and Betty Moore Foundation, the Eileen and Kenneth Norris Foundation, the Caltech Associates, and the National Science Foundation. Ongoing CARMA development and operations are supported by the National Science Foundation under a cooperative agreement, and by the CARMA partner universities.

Facilities: CARMA

\section{REFERENCES}

Allen, D. A., \& Burton, M. G. 1993, Nature, 363, 54

Beckwith, S., Persson, S. E., Neugebauer, G., \& Becklin, E. E. 1978, ApJ, 223, 464
Chandler, C. J., \& de Pree, C. G. 1995, ApJ, 455, L67

Chernin, L. M., \& Wright, M. C. H. 1996, ApJ, 467, 676

Cunningham, N. J., Moeckel, N., \& Bally, J. 2009, ApJ, 692, 943

Doeleman, S. S., Lonsdale, C. J., \& Pelkey, S. 1999, ApJ, 510, L55

Doi, T., O’Dell, C. R., \& Hartigan, P. 2002, AJ, 124, 445

Erickson, N. R., Goldsmith, P. F., Snell, R. L., Berson, R. L., Huguenin, G. R., Ulich, B. L., \& Lada, C. J. 1982, ApJ, 261, L103

Genzel, R., Reid, M. J., Moran, J. M., \& Downes, D. 1981, ApJ, 244, 884

Gezari, D. Y., Backman, D. E., \& Werner, M. W. 1998, ApJ, 509, 283

Gómez, L., Rodríguez, L. F., Loinard, L., Lizano, S., Allen, C., Poveda, A., \& Menten, K. M. 2008, ApJ, 685, 333

Greenhill, L. J., Gwinn, C. R., Schwartz, C., Moran, J. M., \& Diamond, P. J. 1998, Nature, 396, 650

Greenhill, L. J., Reid, M. J., Chandler, C. J., Diamond, P. J., \& Elitzur, M. 2004, in IAU Symp. 221, Star Formation at High Angular Resolution, ed. M. G. Burton, R. Jayawardhana, \& T. L. Bourke (Dordrecht: Kluwer), 155

Kwan, J., \& Scoville, N. 1976, ApJ, 210, L39

Menten, K. M., Reid, M. J., Forbrich, J., \& Brunthaler, A. 2007, A\&A, 474, 515

Plambeck, R. L., Wright, M. C. H., \& Carlstrom, J. E. 1990, ApJ, 348, L65

Reid, M. J., Schneps, M. H., Moran, J. M., Gwinn, C. R., Genzel, R., Downes, D., \& Roennaeng, B. 1988, ApJ, 330, 809

Rodríguez, L. F., Poveda, A., Lizano, S., \& Allen, C. 2005, ApJ, 627, L65

Sandstrom, K. M., Peek, J. E. G., Bower, G. C., Bolatto, A. D., \& Plambeck, R. L. 2007, ApJ, 667, 1161

Stahler, S. W. 1994, ApJ, 422, 616

Stone, J. M., Xu, J., \& Mundy, L. G. 1995, Nature, 377, 315

Terquem, C., Eislöffel, J., Papaloizou, J. C. B., \& Nelson, R. P. 1999, ApJ, 512 L131

Wright, M. C. H., Plambeck, R. L., Mundy, L. G., \& Looney, L. W. 1995, ApJ, 455, L185

Zapata, L. A., Schmid-Burgk, J., Ho, P. T. P., Rodriguez, L. F., \& Menten, K. 2009, ApJL, in press (arXiv:0907.3945v1)

Zuckerman, B., Kuiper, T. B. H., \& Rodriguez Kuiper, E. N. 1976, ApJ, 209, L137 